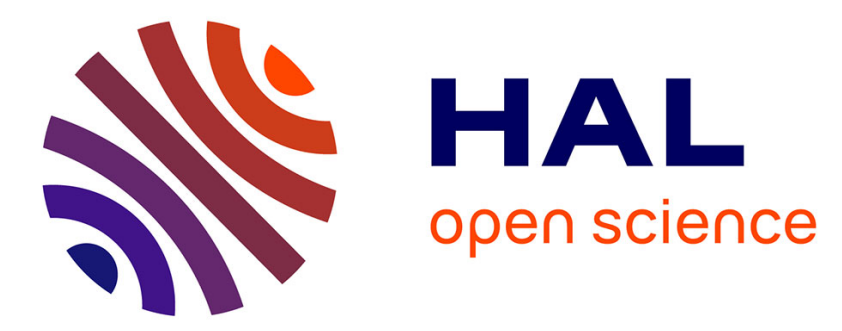

\title{
Mise au point : détermination à partir de la spectroscopie ENDOR de couplages hyperfins dans un radical libre créé dans un cristal organique
}

\author{
P. Gloux
}

\section{- To cite this version:}

P. Gloux. Mise au point: détermination à partir de la spectroscopie ENDOR de couplages hyperfins dans un radical libre créé dans un cristal organique. Revue de Physique Appliquée, 1974, 9 (6), pp.1007-1016. 10.1051/rphysap:01974009060100700 . jpa-00243864

\section{HAL Id: jpa-00243864 https://hal.science/jpa-00243864}

Submitted on 1 Jan 1974

HAL is a multi-disciplinary open access archive for the deposit and dissemination of scientific research documents, whether they are published or not. The documents may come from teaching and research institutions in France or abroad, or from public or private research centers.
L'archive ouverte pluridisciplinaire HAL, est destinée au dépôt et à la diffusion de documents scientifiques de niveau recherche, publiés ou non, émanant des établissements d'enseignement et de recherche français ou étrangers, des laboratoires publics ou privés. 


\title{
MISE AU POINT : DÉTERMINATION A PARTIR DE LA SPECTROSCOPIE ENDOR DE COUPLAGES HYPERFINS DANS UN RADICAL LIBRE CRÉÉ DANS UN CRISTAL ORGANIQUE
}

\author{
P. GLOUX
}

Centre d'Etudes Nucléaires de Grenoble, Département de Recherche Fondamentale, Section de Résonance Magnétique, BP 85, Centre de Tri 38041 Grenoble Cedex, France

(Reçu le 20 mai 1974)

\begin{abstract}
Résumé. - On discute de la détermination par ENDOR des couplages hyperfins pour des radicaux libres créés dans des cristaux organiques. L'exposé est centré sur le cas du couplage hyperfin avec le proton puisque c'est le cas le plus fréquent. Les diverses ambiguïtés que l'on rencontre dans l'identification et le calcul des couplages sont étudiées en détail.

Abstract. - The article discusses the ENDOR determination of the hyperfine couplings of free radicals created in organic crystals. The study examines the case of the hyperfine coupling with the proton which is the common case. The diverse ambiguities that one encounters in the identification and calculation of the couplings are examined in detail.
\end{abstract}

1. Introduction. - La largeur des raies R. P. E. en solide est un handicap considérable dans la recherche des faibles couplages hyperfins puisqu'ils s'y confondent. Aussi, toutes les fois que l'on désire faire une analyse précise d'un radical libre et en particulier dans les cas où le système présente un spin électronique délocalisé, il est nécessaire de recourir à la spectroscopie ENDOR qui donne des raies beaucoup plus fines. Pour les radicaux libres organiques piégés dans des solides, les ordres de grandeur sont sans commune mesure : une largeur de quelques gauss pour les raies R. P. E., une largeur de quelques dizaines de kilocycles pour les raies ENDOR. En outre, même pour des couplages a priori résolus par R.P.E., c'est un avantage considérable que de pouvoir bénéficier de la finesse de la résolution ENDOR, car les défauts créés dans les solides monocristallins obéissent à la symétrie du cristal et il en résulte généralement plusieurs sites qui donnent des spectres R.P. E. mélangés et souvent inextricables. De plus, pour des couplages hyperfins présentant une anisotropie importante, il apparaît les raies R. P. E. correspondant aux transitions dites « interdites ». Et nous ne parlons pas de la superposition fréquente des spectres de plusieurs radicaux au voisinage de la valeur 2,002 3 du facteur spectroscopique $g$ de l'électron libre.

Aussi, bien que les raies ENDOR soient beaucoup plus difficiles à obtenir que les raies R. P. E., l'ENDOR est certainement appelée à supplanter la R.P.E. dans l'étude des radicaux libres dans les cristaux organiques. Toutefois la R.P.E. reste le support nécessaire à l'ENDOR et nous renvoyons le lecteur désireux de s'informer sur le processus de calcul en R. P. E. à l'étude très détaillée de Farach et Poole [1]. Il nous paraît alors utile de faire une mise au point quant à la détermination des couplages par ENDOR : c'est le but de cet article. L'exposé en est divisé en trois parties. Dans la première, nous traitons de manière détaillée du calcul d'un couplage hyperfin supposé isolé. Dans la seconde, l'interaction est replacée dans son contexte, le cristal, et nous examinons les avantages et les inconvénients qui surgissent du fait, en particulier, de l'existence de plusieurs sites et les ambiguités qui peuvent alors survenir dans le calcul. Dans la troisième, qui nous servira de conclusion, nous discutons des problèmes qui se posent encore au stade où l'on rassemble les interactions lors de l'identification du radical. Bien que cette étude soit plus spécifique du domaine des radicaux dans les cristaux organiques, elle pourra par bien des points s'appliquer à d'autres défauts.

2. Calcul d'un couplage hyperfin en ENDOR. 2.1 Hamiltonien. - L'Hamiltonien de spin représentant l'interaction entre l'électron célibataire $\left(S=\frac{1}{2}\right)$ et les divers noyaux doués de spin en présence d'un champ magnétique externe pour un radical libre 
organique qui renferme essentiellement des noyaux de carbone 12 et oxygène $16(I=0)$, azote $14(I=1)$ et hydrogène $1\left(I=\frac{1}{2}\right)$ en abondance naturelle peut être généralement simplifié : en effet les couplages hyperfins de l'azote et du proton sont généralement très faibles (quelques dizaines de $\mathrm{MHz}$ ) par rapport à l'effet Zeeman électronique puisqu'on opère généralement à des champs magnétiques de 3000 et quelques centaines de gauss au moins. L'Hamiltonien de spin total est alors décomposable en une série d'hamiltoniens partiels traitant indépendamment chaque interaction électron-noyau. Ce n'est que dans des cas rares pour ce type de défauts que l'on pourra rencontrer un système obligeant à considérer simultanément plusieurs noyaux, lorsque des couplages presque équivalents sont suffisamment forts [2] (en tout état de cause ce genre de phénomène ne se détectera d'ailleurs qu'en ENDOR : il sera inobservable sur les spectres R. P. E.).

Dans la suite, pour faciliter l'exposé des problèmes que nous développons ici nous nous placerons dans le cas le plus simple et le plus fréquent d'interaction hyperfine dans un radical libre organique, celle entre l'électron et le proton, noyau qui ne présente pas de couplage quadrupolaire à l'inverse de l'azote. $\mathrm{Si}$, d'autre part, comme c'est généralement le cas pour des radicaux organiques, l'anisotropie $\mathrm{du}$ tenseur spectroscopique $\mathbf{g}$ est faible, elle ne rejaillit pas sur les spectres ENDOR et nous pouvons alors considérer que l'hamiltonien exprimé en unité de fréquence est tout simplement :

$$
v_{\mathrm{e}} \mathbf{Z S}+\mathbf{S T I}-v \mathbf{Z I} \text {. }
$$

Le champ magnétique est dirigé suivant la direction Z. Il lui correspond $v_{\mathrm{e}}$ et $v$, les fréquences de résonance dans ce champ de l'électron et du proton libres: pour un champ magnétique de 3000 et quelques centaines de gauss, elles sont autour respectivement de $9 \mathrm{GHz}$ et $14 \mathrm{MHz}$. T est le tenseur symétrique de second rang de l'interaction hyperfine entre les spins $\mathbf{S}$ et $\mathbf{I}$ de l'électron et du proton.

Nous sommes dans le cas où $v_{\mathrm{e}} \mathbf{Z S} \gg \mathbf{S T I}-v \mathbf{Z I}$. $v_{\mathrm{e}} \mathbf{Z S}$ est l'ordre 0 d'une méthode de perturbation et nous supposons qu'il suffit de traiter le reste au premier ordre.

A l'ordre 0 le spin électronique est quantifié dans la direction $\mathbf{Z}$ pour les valeurs propres $\pm \frac{1}{2}$ de $S_{z}$,

A l'ordre 1, suivant que l'on se trouve dans l'un ou l'autre des états propres de $v_{\mathrm{e}} \mathrm{ZS}$, la perturbation est équivalente aux opérateurs

$$
-\mathbf{Z}\left(\mp \frac{\mathbf{T}}{2}+v\right) \mathbf{I} \text { pour } S_{z}= \pm \frac{1}{2}
$$

qui diagonalisés correspondent à la quantification de I suivant la direction

$$
\mathbf{Z}^{\prime}=\mathbf{Z}\left(-\frac{\mathbf{T}}{2}+v\right)
$$

pour les valeurs propres $\mp \frac{1}{2}$ de $I_{z^{\prime}}$ ou suivant la direction

$$
\mathbf{Z}^{\prime \prime}=\mathbf{Z}\left(\frac{\mathbf{T}}{2}+v\right)
$$

pour les valeurs propres $\mp$ de $I_{z^{\prime \prime}}$.

Les niveaux sont donc:

$$
\begin{gathered}
\frac{v_{\mathrm{e}}}{2}+\frac{1}{2}\left|\mathbf{Z}^{\prime}\right| \\
\frac{v_{\mathrm{e}}}{2}-\frac{1}{2}\left|\mathbf{Z}^{\prime}\right| \\
-\frac{v_{\mathrm{e}}}{2}+\frac{1}{2}\left|\mathbf{Z}^{\prime \prime}\right| \\
-\frac{v_{\mathrm{e}}}{2}-\frac{1}{2}\left|\mathbf{Z}^{\prime \prime}\right| .
\end{gathered}
$$

Il leur correspond les transitions ENDOR $\left(\Delta M_{\mathrm{s}}=0\right.$, $\left.\Delta m_{I}=1\right)\left|\mathbf{Z}^{\prime}\right|$ ou $\left|\mathbf{Z}^{\prime \prime}\right|$. Pour ce système, dans le cas où $v$ est plus petit que $|\mathbf{Z T} / 2|$, la mesure de l'écart entre les raies R. P. E. correspond à $\left|\mathbf{Z}^{\prime}\right|+\left|\mathbf{Z}^{\prime \prime}\right|$ entre les transitions permises $\left(\Delta M_{\mathrm{s}}=1, \Delta m_{I}=0\right)$ et || $\mathbf{Z}^{\prime}|-| \mathbf{Z}^{\prime \prime}||$ entre les transitions interdites

$$
\left(\Delta M_{\mathrm{s}}=1, \Delta m_{I}=1\right) ;
$$

et vice versa pour $v>|\mathbf{Z T} / 2|$.

Nous notons donc ici encore une supériorité de l'ENDOR sur la R.P.E.: le module représentant la transition ENDOR est une expression beaucoup plus maniable que la somme ou la différence des modules donnée par la R.P. E. Nous verrons plus loin que la transition ENDOR est directement reliée à un élément d'un tenseur carré, ce n'est évidemment pas le cas pour l'écart entre les transitions " permises» obtenu par R. P. E. : ce n'est que lorsqu'on détecte aussi les transitions "interdites" que la R.P.E. est équivalente à l'ENDOR. Ainsi les deux raies ENDOR fournissent autant de renseignements que l'ensemble des quatre raies R. P. E.

Pour en rester dans le domaine des comparaisons, nous noterons encore qu'aux hamiltoniens indépendants liés aux divers noyaux correspondent des transitions ENDOR elles aussi indépendantes, ce qui n'est pas le cas des transitions R.P.E. correspondantes qui font intervenir des sommes ou des différences des différents modules liés aux divers couplages. Il faut donc dans le spectre R.P.E. rechercher les raies entre lesquelles se mesure la somme ou la différence des modules pour un couplage dònné, alors que les raies ENDOR sont analysées de manière indépendante pour les différents couplages. Notons aussi que le nombre de raies ENDOR est bien moindre que celui de raies R. P. E. «permises » : par exemple respectivement $2 n$ et $2^{n}$ pour $n$ couplages inéquivalents avec des noyaux de spin $\frac{1}{2}$.

2.2 CALCUl DU Tenseur hyperfin T. - On ne sait pas attribuer l'une et l'autre des transitions 
ENDOR expérimentales obtenues dans une direction $\mathbf{Z}$ du champ magnétique à la valeur propre correspondante du spin électronique. Nous ne pouvons que représenter arbitrairement l'une d'entre elles, appelée $m_{+}$, par l'expression $|\mathbf{Z}(\mathbf{X}+v)|$ et en conséquence l'autre, $m_{-}$, par $|\mathbf{Z}(-\mathbf{X}+v)|$. A la fin du calcul nous obtenons le tenseur $\mathbf{T}$ par $\mathbf{T}= \pm 2 \mathbf{X}$ avec une indétermination globale sur les signes de ses éléments. Ce n'est que par la comparaison du tenseur expérimental $\mathbf{T}$ avec celui correspondant à un modèle théorique adéquat, en liaison avec la structure du radical, et la chimie quantique, qu'il sera ultérieurement possible de lever cette ambiguïté.

Nous allons donc déterminer $\mathbf{X}$. Nous posons $\mathbf{X}+v=\mathbf{A}_{v+}$ et $-\mathbf{X}+v=\mathbf{A}_{v-}$. De $m_{+}=\left|\mathbf{Z} \mathbf{A}_{v+}\right|$ on déduit $m_{+}^{2}=\mathbf{Z A}_{v+}^{2} \mathbf{Z}$. De même $m_{-}^{2}=\mathbf{Z A}_{v-}^{2} \mathbf{Z}$. Le carré d'une transition ENDOR est donc simplement la composante dans la direction $\mathbf{Z}$ d'un tenseur carré. Il est alors aisé d'obtenir, à partir d'un certain nombre d'orientations du champ magnétique dans l'espace, l'expression matricielle de $\mathbf{A}_{v+}^{2}$ ou de $\mathbf{A}_{v-}^{2}$ dans un repère déterminé. Il y a ici une petite diffculté : nous avons implicitement supposé que $v$ était le même dans toutes les directions de l'espace pour pouvoir définir l'un ou l'autre des tenseurs qui le renferment. Dans la pratique, il n'en est pas ainsi car la grandeur du champ magnétique va dépendre de sa direction (et cela même si l'on réussit à maintenir constante la fréquence du champ hyperfréquence). Les raies ENDOR correspondent à une variation dans l'intensité du signal R. P. E. (préalablement saturé) : elles seront d'autant plus grandes que le signal R. P. E. est initialement plus intense, ce qui nécessitera d'opérer au niveau d'un extremum du signal R.P.E. En raison de l'anisotropie du tenseur $\mathbf{g}$, mais surtout de l'anisotropie des couplages hyperfins, il sera donc généralement nécessaire de constamment réajuster la valeur du champ en fonction de sa direction pour le maintenir au niveau d'un extremum du signal R. P. E. Néanmoins, pour des cristaux organiques le tenseur $\mathbf{g}$ et les couplages hyperfins sont généralement suffisamment peu anisotropes pour que la variation de $v$ reste faible. Cela permettra généralement d'utiliser dans un calcul préliminaire destiné à éliminer les ambiguïtés qui vont apparaître par la suite une valeur moyenne de $v$, avant de procéder dans le calcul définitif à des corrections précises sur la valeur des transitions afin de les référer à un même $v$ pour les différentes orientations du champ utilisées dans le calcul.

Dans un repère orthonormé donné, l'un des tenseurs, appelons-le $\mathbf{A}_{v}^{2}$, sera exprimé par une matrice $3 \times 3$ symétrique qui, une fois diagonalisée, sera représentée par ses valeurs et ses vecteurs propres. La connaissance du tenseur nécessite donc le calcul de six paramètres indépendants, ce qui revient à déterminer les transitions dans six directions de l'espace dont trois au plus sont dans un même plan. Dans le repère abc les trois termes diagonaux $\mathrm{aA}_{v}^{2} \mathbf{a}$, $\mathbf{b} \mathbf{A}_{v}^{2} \mathbf{b}, \mathbf{c A _ { v } ^ { 2 }} \mathbf{c}$ sont immédiatement identifiés à $m_{\mathbf{a}}^{2}, m_{\mathbf{b}}^{2}$, $m_{\mathrm{c}}^{2}$ et le plus simple est de calculer chaque terme non diagonal $\mathbf{i} \mathbf{A}_{v}^{2} \mathbf{j}$ à partir de transitions observées dans des directions appartenant au plan $i j$. Nous calculons par exemple $\mathbf{a} \mathbf{A}_{v}^{2} \mathbf{b}$ par ce qui suit :

Dans une direction $\mathbf{Z}$ du plan $a b$, avec $\theta=(\mathbf{a}, \mathbf{Z})$, on a

$$
\begin{aligned}
& m^{2}(\theta)=\mathbf{Z A}_{v}^{2} \mathbf{Z}=\|\cos \theta \sin \theta\| \times \\
& \times\left\|\begin{array}{ll}
\mathbf{a} \mathbf{A}_{v}^{2} \mathbf{a} & \mathbf{a} \mathbf{A}_{v}^{2} \mathbf{b} \\
\mathbf{a} \mathbf{A}_{v}^{2} \mathbf{b} & \mathbf{b} \mathbf{A}_{v}^{2} \mathbf{b}
\end{array}\right\|\left\|\begin{array}{c}
\cos \theta \\
\sin \theta
\end{array}\right\| \\
& =\frac{1}{2}\left(\mathbf{a} \mathbf{A}_{v}^{2} \mathbf{a}-\mathbf{b} \mathbf{A}_{v}^{2} \mathbf{b}\right) \cos 2 \theta \\
& +\mathbf{a} \mathbf{A}_{v}^{2} \mathbf{b} \sin 2 \theta+\frac{1}{2}\left(\mathbf{a} \mathbf{A}_{v}^{2} \mathbf{a}+\mathbf{b} \mathbf{A}_{v}^{2} \mathbf{b}\right) .
\end{aligned}
$$

La courbe $m^{2}(\theta)$ est une sinusoïde (de période $\pi$ ) : elle est complètement déterminée à partir de trois transitions.

Soit encore

$$
\begin{aligned}
m^{2}(\theta)=\frac{1}{2}\left(m_{\mathbf{a}}^{2}-m_{\mathbf{b}}^{2}\right) & \cos 2 \theta+ \\
& +\mathbf{a} \mathbf{A}_{v}^{2} \mathbf{b} \sin 2 \theta+\frac{1}{2}\left(m_{\mathbf{a}}^{2}+m_{\mathbf{b}}^{2}\right)
\end{aligned}
$$

qui permet de calculer $\mathbf{a} \mathbf{A}_{v}^{2} \mathbf{b}$ à partir de $m_{\mathbf{a}}^{2}, m_{\mathbf{b}}^{2}$ et un autre $m^{2}(\theta)$.

On pourra utiliser les relations suivantes :

$$
\begin{aligned}
& \mathbf{a} \mathbf{A}_{v}^{2} \mathbf{b}=m^{2}\left(\frac{\pi}{4}\right)-\frac{1}{2}\left(m_{\mathbf{a}}^{2}+m_{\mathbf{b}}^{2}\right) \\
&=\frac{1}{2}\left(m_{\mathbf{a}}^{2}+m_{\mathbf{b}}^{2}\right)-m^{2}\left(\frac{3 \pi}{4}\right) \\
&=\frac{1}{2}\left[m^{2}\left(\frac{\pi}{4}\right)-m^{2}\left(\frac{3 \pi}{4}\right)\right] \\
&\left(\mathbf{a} \mathbf{A}_{v}^{2} \mathbf{b}\right)^{2}=\frac{1}{4}\left[\left(m_{\max }^{2}-m_{\min }^{2}\right)^{2}-\left(m_{\mathbf{a}}^{2}-m_{\mathbf{b}}^{2}\right)^{2}\right] .
\end{aligned}
$$

Bien que mathématiquement les six transitions qui définissent le tenseur, à raison de trois ou plus dans un même plan, pourraient correspondre à des directions très rapprochées, il est clair que la précision dans la pratique est obtenue à partir de transitions suffisamment disséminées dans les directions de l'espace. De ce point de vue, l'utilisation de transitions dans les directions $\mathbf{a}, \mathbf{b}, \mathbf{c}$ et leurs directions bissectrices s'impose. Une meilleure précision sera bien sûr obtenue en utilisant un plus grand nombre de transitions, ce qui permettra d'appliquer une méthode des moindres carrés.

La diagonalisation de la matrice de $\mathbf{A}_{v}^{2}$ nous donne ses vecteurs propres dans le repère $\mathbf{a}, \mathbf{b}$, $\mathbf{c}$ pour les valeurs propres $P_{1}, P_{2}, P_{3}$. Il en résulte huit tenseurs $\mathbf{A}_{v}$ possibles de mêmes vecteurs propres mais 
dont les valeurs propres sont différenciées par le signe :

$$
\pm\left(P_{1}\right)^{1 / 2}, \pm\left(P_{2}\right)^{1 / 2}, \pm\left(P_{3}\right)^{1 / 2}
$$

Il en est de même pour $\mathbf{X}$ et, par exemple à partir de $\mathbf{A}_{v+}^{2}$, les huit solutions sont exprimées par les valeurs propres :

$$
\begin{gathered}
X_{1}=-v \pm\left(P_{1+}\right)^{1 / 2}, \quad X_{2}=-v \pm\left(P_{2+}\right)^{1 / 2}, \\
X_{3}=-v \pm\left(P_{3+}\right)^{1 / 2} .
\end{gathered}
$$

De même à partir de $\mathbf{A}_{v}^{2}$ on obtient :

$$
\begin{gathered}
X_{1}=v \pm\left(P_{1-}\right)^{1 / 2}, \quad X_{2}=v \pm\left(P_{2-}\right)^{1 / 2}, \\
X_{3}=v \pm\left(P_{3-}\right)^{1 / 2} .
\end{gathered}
$$

Bien entendu une seule solution sera commune à l'une et l'autre des deux séries et ce sera la solution du problème physique recherchée.

En fait, on accède plus rapidement à cette solution commune. Puisque

$$
(\mathbf{X}+v)^{2}=\mathbf{A}_{v+}^{2} \quad \text { et }(-\mathbf{X}+v)^{2}=\mathbf{A}_{v-}^{2},
$$

il en résulte la relation

$$
\mathbf{X}=\frac{1}{4 v}\left(\mathbf{A}_{v+}^{2}-\mathbf{A}_{v-}^{2}\right)
$$

qui fournit immédiatement la matrice de $\mathbf{X}$ dans le repère abc. Il suffit de remplacer, pour la détermination des éléments $\mathbf{a} \mathbf{X} \mathbf{a}, \ldots, \mathbf{a} \mathbf{X} \mathbf{b}, \ldots$, les éléments $\mathbf{a} \mathbf{A}_{v}^{2} \mathbf{a} \ldots$, $\mathbf{a} \mathbf{A}_{v}^{2} \mathbf{b} \ldots$ par leurs expressions en fonction des transitions indiquées ci-dessus.

On peut théoriquement calculer tous les éléments $\mathrm{du}$ tenseur $\mathbf{X}$, mais certains seulement en valeur absolue, à l'aide d'un nombre moindre de directions où l'on détecte les deux espèces de transitions. Ainsi, à partir de transitions dans les directions des trois axes $\mathbf{a}, \mathbf{b}, \mathbf{c}$ on connaît $\mathbf{a} \mathbf{X} \mathbf{a}, \mathbf{b} \mathbf{X} \mathbf{b}, \mathbf{c} \mathbf{X} \mathbf{c}$ comme ci-dessus. On peut alors les reporter dans les expressions donnant les transitions par exemple $m_{+}$dans les directions $\mathbf{a}, \mathbf{b}, \mathbf{c}$ en fonction des éléments du tenseur $\mathbf{X}$. Ce qui conduit à :

$$
\begin{aligned}
& A=m_{\mathbf{a}+}^{2}-(\mathbf{a} \mathbf{X} \mathbf{a}+v)^{2}=(\mathbf{a} \mathbf{X} \mathbf{b})^{2}+(\mathbf{a} \mathbf{X} \mathbf{c})^{2} \\
& B=m_{\mathbf{b}+}^{2}-(\mathbf{b} \mathbf{X} \mathbf{b}+v)^{2}=(\mathbf{a} \mathbf{X} \mathbf{b})^{2}+(\mathbf{b} \mathbf{X} \mathbf{c})^{2} \\
& C=m_{\mathbf{c}+}^{2}-(\mathbf{c} \mathbf{X} \mathbf{c}+v)^{2}=(\mathbf{a} \mathbf{X} \mathbf{c})^{2}+(\mathbf{b} \mathbf{X} \mathbf{c})^{2} .
\end{aligned}
$$

On en déduit :

$$
\begin{aligned}
& |\mathbf{a} \quad \mathbf{b}|=\left(\frac{A+B-C}{2}\right)^{1 / 2} \\
& |\mathbf{b} \mathbf{X} \mathbf{c}|=\left(\frac{B+C-A}{2}\right)^{1 / 2} \\
& |\mathbf{a} \mathbf{X} \mathbf{c}|=\left(\frac{A+C-B}{2}\right)^{1 / 2} \text {. }
\end{aligned}
$$

Toutefois, les termes non diagonaux $(\mathbf{a} \mathbf{X} \mathbf{b}, \ldots)$ sont généralement faibles par rapport aux termes dia- gonaux $(\mathbf{a} \mathbf{X} \mathbf{a}+v, \ldots)$. Il en résulte que dans ce calcul l'erreur sur la transition a beaucoup d'influence sur les termes non diagonaux. En fait on aura éventuellement recours à ce genre de calcul seulement dans le cas où, pour une interaction hyperfine, on ne détecte dans un plan, par exemple $a b$, que les transitions dans les directions des axes $\mathbf{a}$ et $\mathbf{b}$ : cela permettra de se faire une idée de la valeur du terme non diagonal correspondant.

Lorsque l'on possède seulement une espèce de transitions qu'arbitrairement nous identifions par exemple aux transitions + , s'il est possible de se placer à deux fréquences de résonance du proton libre différentes, et ce sera le cas si l'on peut obtenir les raies ENDOR de même espèce dans deux régions différentes du spectre R.P.E., il est mathématiquement possible d'obtenir $\mathbf{X}$ par l'équation :

$$
2 \mathbf{X}=\frac{1}{v^{\prime}-v}\left(\mathbf{A}_{v^{\prime}+}^{2}-\mathbf{A}_{v+}^{2}\right)-\left(v^{\prime}+v\right) .
$$

Dans la pratique, l'écart entre les deux fréquences sera généralement faible en raison du peu d'étendue du spectre R.P.E. On serait donc conduit à trop d'imprécision sur le tenseur $\mathbf{X}$ obtenu par cette relation et on ne l'utilisera alors qu'afin de choisir, dans la mesure du possible, entre les huit solutions.

Une fois trouvée la bonne solution $\mathbf{X}$, c'est cette dernière relation pour les transitions + (ou celle équivalente pour les transitions -) que l'on utilise pour déterminer la correction sur la transition lorsque l'on ramène la fréquence de résonance du proton libre à une valeur commune dans les directions concernées par le calcul définitif de $\mathbf{X}$. On peut aussi l'exprimer sous une forme différentielle : à partir de

$$
m_{+}^{2}=\mathbf{Z}(\mathbf{X}+v)^{2} \mathbf{Z}
$$

on obtient immédiatement

$$
\mathrm{d} m_{+}=\frac{\mathbf{Z X Z}+v}{m_{+}} \mathrm{d} v
$$

et de même

$$
\mathrm{d} m_{-}=\frac{-\mathbf{Z} \mathbf{X Z}+v}{m_{-}} \mathrm{d} v
$$

Nous noterons qu'il peut aussi se faire que des informations déduites de l'écart entre des raies R. P. E. contribuent à trouver la bonne solution $\mathbf{X}$. Si toutefois il reste une quelconque ambiguïté à ce niveau, on peut espérer ultérieurement utiliser la comparaison avec le modèle théorique adéquat, comme lors de la détermination du signe absolu de $\mathbf{T}$.

2.3 ANISOTROPIE DU TENSEUR HYPERFIN. - Nous nous intéressons maintenant à quelques questions en rapport avec l'anisotropie du tenseur $\mathbf{T}$ (ou du tenseur $\mathbf{X}= \pm \mathbf{T} / 2$ )

Il faut prendre garde à ne pas assimiler l'anisotropie du tenseur $\mathbf{T}$ à l'anisotropie présentée par l'une ou 
l'autre espèce des transitions ENDOR correspondantes. L'anisotropie du tenseur $\mathbf{T}$ correspond à la variation de $\mathbf{Z T Z}$ en fonction de l'orientation. Comme nous l'avons vu, $\mathbf{Z T Z}$ est proportionnel à la différence entre les carrés des transitions + et - obtenues dans la direction $\mathbf{Z}$ et sa variation en fonction de l'orientation dans un plan sera donc visualisée par l'écart séparant les deux courbes $m_{+}^{2}(\theta)$ et $m_{-}^{2}(\theta)$. Par contre l'une de ces courbes représente la variation de l'élément positif $\mathbf{Z A}_{v}^{2} \mathbf{Z}$ du tenseur carré $\mathbf{A}_{v}^{2}$ correspondant. Ainsi le tenseur $\mathbf{T}$ peut a priori renfermer une anisotropie plus importante que ne le laisserait soupçonner la vue de l'une seulement de ces courbes.

Les deux transitions ENDOR dans une direction $\mathbf{Z}$ sont égales aux modules des deux diagonales du parallélogramme formé par les vecteurs $\mathbf{Z X}$ et $v \mathbf{Z}$. Lorsque c'est un rectangle (quand $\mathbf{Z X}$ est perpendiculaire à $v \mathbf{Z}$ ), les modules et donc les transitions sont égaux : dans un plan contenant $\mathbf{Z}$, les courbes ENDOR + et - se coupent suivant cette direction à travers laquelle $\mathbf{Z} \mathbf{X Z}$ change de signe. Nous noterons que cette égalité ne peut arriver que pour une valeur de $m$ supérieure à $v$.

Par ailleurs, en particulier dans les interactions avec des protons, on peut fréquemment rencontrer des tenseurs $\mathbf{T}$ présentant une anisotropie suffisamment faible par rapport à l'effet isotrope total (où sont impliquées la partie isotrope du tenseur et la fréquence de résonance $v$ du proton libre) pour que les termes non diagonaux $\mathbf{Z X A}$ et $\mathbf{Z X B}$ (où $\mathbf{A}$ et $\mathbf{B}$ forment un trièdre trirectangle avec $\mathbf{Z}$ ) aient une faible influence dans toute direction $\mathbf{Z}$ : la direction de $\mathbf{Z X}$ est alors constamment proche de celle de $\mathbf{Z}$. Alors

$$
m_{+}^{2}=\mathbf{Z}(\mathbf{X}+v)^{2} \mathbf{Z} \#(\mathbf{Z} \mathbf{X Z}+v)^{2}
$$

et de même

$$
m_{-}^{2} \#(-\mathbf{Z} \mathbf{X Z}+v)^{2}
$$

On a donc :

$$
m_{+} \#|\mathbf{Z} \mathbf{X Z}+v| \text { et } m_{-} \#|-\mathbf{Z} \mathbf{X} \mathbf{Z}+v|
$$

et il en résulte que :

$$
\left|\frac{\mathrm{d} m_{ \pm}}{\mathrm{d} v}\right| \# 1 .
$$

On peut distinguer alors entre deux cas :

a) $\mathrm{Si} v$ a une influence plus grande que $\mathbf{X}$, les courbes $m_{+}(\theta)$ et $m_{-}(\theta)$ de tout plan sont plus ou moins symétriques par rapport à la droite représentant $v$. On a

$$
\frac{\mathrm{d} m_{ \pm}}{\mathrm{d} v} \not 1
$$

et ainsi pour une variation $\Delta v$ de $v$ l'ensemble des deux courbes et de la droite est translaté de $\Delta v$.

b) Si la partie isotrope de $\mathbf{X}$ a une influence prépondérante, $\mathbf{Z X Z}$ conserve toujours le même signe et les deux courbes sont séparées par une distance de l'ordre de $2 v$. Pour la plus élevée en fréquence on a

$$
\frac{\mathrm{d} m}{\mathrm{~d} v} \# 1
$$

et pour la plus basse on a

$$
\frac{\mathrm{d} m}{\mathrm{~d} v} \#-1 \text {. }
$$

Lorsque $v$ croît, les courbes s'éloignent l'une de l'autre de $2 \Delta v$.

3. Problèmes liés au cristal, plus spécialement organique, dans la détermination d'un couplage hyperfin par ENDOR. - 3.1 PROBLÈMES EXPÉRIMENTAUX. Pour calculer un tenseur hyperfin, il suffit d'obtenir les transitions ENDOR correspondantes dans six directions (dont trois au plus dans un même plan); pour obtenir la matrice du tenseur dans un repère orthonormé abc la solution la plus directe consiste à utiliser les transitions dans les directions des axes abc et de leurs trois bissectrices intérieures par exemple. Mais en fait, si la détection des raies ENDOR était limitée à quelques directions de l'espace il ne serait généralement pas possible d'identifier celles d'une interaction hyperfine de celles des autres; de plus, nous verrons ci-dessous que le radical présente généralement plusieurs sites, ce qui ne peut que compliquer le spectre ENDOR puisque bien souvent les raies R. P. E. des différents sites se chevauchent et que l'on détecte ainsi simultanément leurs raies ENDOR. Il sera donc généralement nécessaire d'effectuer un balayage des trois plans $a b, b c$ et $c a$ par la direction du champ magnétique de façon à identifier, pour chaque interaction, les courbes des transitions ENDOR dans les trois plans.

Après ce préliminaire, nous allons maintenant examiner les conséquences qu'implique au niveau de la détermination de l'interaction hyperfine l'existence de sites moléculaires dans le cristal. Nous savons, les études par R. P. E. de radicaux dans les cristaux organiques le prouvent, qu'il en est de même pour le radical.

Le nombre de sites qui restent magnétiquement inéquivalents, que nous appellerons ici sites hyperfins, est égal au nombre d'éléments que renferme le groupe des rotations propres du cristal. Toutefois, ce nombre serait diminué si le radical était invariant dans les opérations de symétrie d'un sous-groupe de ce groupe de telle sorte que l'interaction hyperfine le soit elle aussi (un autre cas serait qu'une interaction hyperfine se transforme en une autre lors de l'invariance du radical). On peut se reporter aux articles de Rae [3] ou de Weil et al. [4] pour les problèmes de symétries et de sites, d'un point de vue magnétique. En particulier, Rae indique le nombre de sites magnétiquement inéquivalents à attendre suivant la direction pour chacun des onze groupes de rotations propres 
qui peuvent exister en cristal et présente les différents cas de figures.

L'existence de sites hyperfins devrait théoriquement faciliter grandement l'obtention du résultat en réduisant le nombre des plans à étudier nécessaire pour déterminer le tenseur. En effet, pour une interaction hyperfine, l'existence de $x$ sites hyperfins donne généralement dans un plan quelconque $x$ couples de courbes ENDOR + et - : on visualise ainsi en un seul plan les courbes d'un site hyperfin appartenant à $x$ plans, qui sont identifiables en appliquant les symétries du groupe des rotations concerné au seul plan étudié ; dans la mesure où $x$ est $\geqslant 3$, le tenseur est alors calculable (à partir d'expressions un peu moins simples que celles indiquées ci-dessus puisque les $x$ plans ne seront généralement pas perpendiculaires entre eux). L'article de Weil et al. [4] fournit une étude très détaillée de la question. Le nombre de sites hyperfins sera $\geqslant 3$ pour 9 des 11 groupes de rotations propres ; seuls font exception les deux groupes respectivement du système triclinique et du système monoclinique.

Nous devons alors remarquer que la complexité des molécules organiques fait que la majorité des cristaux organiques appartient aux systèmes cristallins de basse symétrie [5]: triclinique, monoclinique et orthorhombique. Le système triclinique présente un seul site hyperfin (l'identité est le seul élément du groupe des rotations) et nécessite de toute manière trois plans d'étude. Le système monoclinique $(2$ sites hyperfins : un axe binaire définit le groupe des rotations propres) nécessiterait deux plans d'étude dont l'un au moins ne passerait pas par l'axe binaire. Le système orthorhombique (4 sites hyperfins : trois axes binaires perpendiculaires entre eux définissent le groupe des rotations propres) nécessiterait seulement un plan d'étude ne passant pas par les axes binaires.

Bien entendu, nous aurons intérêt, chaque fois que cela est possible, à n'utiliser qu'un ou deux plans d'étude pour les systèmes respectivement orthorhombique et monoclinique. Dans la pratique, malheureusement, diverses raisons font que dans l'étude ENDOR de cristaux organiques présentant ces deux types de symétrie, il est souvent préférable, voire nécessaire, d'utiliser les transitions obtenues dans trois plans particuliers perpendiculaires entre eux. Nous allons maintenant examiner ces raisons et simultanément définir ces plans.

Comme tout cristal, un cristal organique se développe macroscopiquement suivant certaines directions privilégiées. Les faces externes pour un cristal obtenu par évaporation lente d'une solution saturée ou les faces obtenues par clivage pour un cristal élaboré par une technique de fusion de zones correspondent à des plans très particuliers en relation géométrique simple avec les directions des axes de rotations propres. Généralement dans la préparation de l'expérience ENDOR, on est alors naturellement conduit à coller le cristal sur le porte-échantillon par une face externe renfermant la direction de l'axe binaire (cas du système monoclinique) ou renfermant les directions de deux axes binaires (cas du système orthorhombique). Cette face pourra être placée suivant trois positions de telle sorte que le champ magnétique balaie successivement les trois plans perpendiculaires entre eux dont les intersections sont les axes binaires (cas du système orthorhombique), deux plans perpendiculaires entre eux d'intersection l'axe binaire et le plan perpendiculaire à l'axe (cas du système monoclinique). On obtiendra, d'autre part, une grande précision dans la définition des plans sous considération par rapport au champ magnétique en utilisant le fait que dans la direction des axes binaires tous les sites hyperfins deviennent équivalents et par conséquent leurs raies ENDOR, pour une interaction hyperfine donnée, se confondent; en raison de la finesse des raies ENDOR on atteint facilement une précision inférieure au degré dans la coïncidence du champ magnétique et d'un axe, coïncidence qui sera réalisée par retouches successives de l'orientation du cristal sur le porte-échantillon.

Par contre, travailler dans un plan quelconque poserait des problèmes importants. Outre le fait qu'une bonne identification de ce plan serait difficile à obtenir, il faudrait généralement se livrer à une opération délicate pour des cristaux organiques, éventuellement petits et souvent facilement friables : la taille des cristaux. D'autre part, même si dans les expériences d'ENDOR on ne peut généralement utiliser que des échantillons relativement petits (quelques $\mathrm{mm}$ ) entre autres pour des raisons d'encombrement, il est toutefois primordial d'occuper au maximum le volume permis pour augmenter les possibilités de détection des raies ENDOR: la taille des cristaux risque d'aller à l'encontre de cette exigence.

Il y a enfin dans le cas du système orthorhombique, dans le souci encore d'arriver à détecter le signal ENDOR, une autre raison parfois vitale de travailler dans les plans privilégiés. C'est que, alors que dans une direction quelconque on compte généralement quatre sites hyperfins, dans les directions des plans privilégiés on en compte seulement deux (et même un seul dans la direction des axes binaires). Toutes les fois que l'on détecte avec difficulté les courbes ENDOR, il sera important de gagner de la sorte un facteur 2 sur le rapport signal/bruit. Enfin, en présence de nombreuses interactions hyperfines, dans le cas où l'on détecte simultanément les raies ENDOR des différents sites hyperfins, il peut parfois être intéressant de travailler avec un nombre de sites restreint pour arriver à suivre les courbes ENDOR. Remarquons au passage que pour des symétries plus élevées, à plus grand nombre de sites hyperfins, les problèmes de la perte d'intensité ENDOR et de la confusion des spectres seraient encore plus cruciaux.

3.2 Ambiguïtés dans le Calcul. - Tant dans le cas du système monoclinique que dans celui du sys- 
tème orthorhombique, le calcul d'un tenseur d'interaction hyperfine renferme une ambiguïté qui provient de la symétrie binaire, lorsque l'étude ENDOR est réalisée dans les trois plans privilégiés définis plus haut. Nous allons voir que si l'on ne dispose que d'une seule espèce de transitions pour un $v$ donné, il en résultera généralement pour les différents sites hyperfins deux séries de solutions tensorielles possibles entre lesquelles le choix ne pourra être fait par le calcul. Par contre, nous verrons que si l'on dispose des deux espèces de transitions, l'ambiguïté sera aisément résolue.

Dans la suite de ce paragraphe nous ne préciserons pas à chaque fois que nous utilisons une seule espèce de transitions ENDOR pour une interaction hyperfine donnée : c'est seulement au dernier sous-paragraphe qu'interviennent les deux espèces de transitions ENDOR pour l'interaction hyperfine étudiée.

Les transitions ENDOR appartenant à deux sites hyperfins liés par symétrie par rapport à un axe binaire se correspondent dans cette symétrie. Dans le cas du système monoclinique, dans un plan contenant l'axe, les courbes ENDOR des deux sites sont alors symétriques par rapport à cet axe et du fait de leur périodicité de $\pi$ sont aussi symétriques par rapport à la perpendiculaire à cet axe; dans le plan perpendiculaire à l'axe, la symétrie, alliée à la périodicité de $\pi$, font que les courbes sont confondues. Dans le cas du système orthorhombique, où les quatre sites hyperfins sont mutuellement liés par symétrie par rapport aux trois axes binaires, il n'existe que deux courbes distinctes dans chacun des trois plans, symétriques l'une de l'autre par rapport aux axes binaires de ce plan : les sites sont deux à deux équivalents dans chaque plan par permutation circulaire. En outre, la symétrie impose l'équivalence de tous les sites dans les directions intersections des trois plans puisque ce sont la direction de l'axe binaire et deux directions perpendiculaires à cet axe (cas du système monoclinique), les directions des axes binaires (cas du système orthorhombique) : les courbes se coupent donc dans ces directions.

A deux sites hyperfins symétriques l'un de l'autre par rapport à un axe binaire correspondent des tenseurs dont les matrices dans le repère abc constitué par les directions intersections des trois plans, se différencient seulement par le changement de signe des deux éléments non diagonaux liés à cet axe binaire. La diagonalisation fournit des valeurs principales identiques et des directions principales qui se distinguent par le changement du signe de chacun des trois cosinus directeurs relatifs à cet axe.

L'ambiguïté réside dans le fait qu'a priori on ne sait comment associer les courbes, d'un plan à un autre, pour qu'elles constituent celles d'un site hyperfin. Le système monoclinique présente quatre possibilités d'association pour seulement deux sites : autrement dit un site étant caractérisé par la courbe unique du plan perpendiculaire à l'axe et l'une des deux courbes d'un autre plan, il faut identifier dans le troisième plan la courbe qui lui correspond. Le système orthorhombique présente huit possibilités d'association pour seulement quatre sites : un site étant caractérisé par une courbe d'un plan et une autre d'un deuxième plan, il faut prendre garde à correctement choisir la courbe du troisième plan. Dans chaque cas, il en résultera généralement deux séries distinctes de solutions possibles pour les différents sites.

Concrètement, pour le site hyperfin que nous venons de caractériser, l'ambiguïté revient à ignorer le signe du terme non diagonal lié au troisième plan dans la matrice du tenseur carré. La partie purement dipolaire de ce tenseur, en tenant compte de l'alternative sur le signe, est alors représentée dans le repère abc par:

$$
\left\|\begin{array}{ccc}
a & d & e \\
d & b & \pm f \\
e & \pm f & c
\end{array}\right\|
$$

L'équation aux valeurs propres est :

$$
\begin{aligned}
x^{3}+x(b c+a b & \left.+a c-f^{2}-d^{2}-e^{2}\right)- \\
& -a b c \mp 2 d e f+a f^{2}+c d^{2}+b e^{2}=0
\end{aligned}
$$

qui a trois racines réelles si :

$$
\begin{aligned}
4\left(b c+a b+a c-f^{2}-d^{2}-e^{2}\right)^{3}+ \\
+27\left(a f^{2}+c d^{2}+b e^{2}-a b c \mp 2 d e f\right)^{2} \leqslant 0 .
\end{aligned}
$$

Puisque les tenseurs hyperfins dans les cristaux organiques sont a priori quelconques par rapport au repère abc, les éléments non diagonaux sont généralement tous non nuls, ce qui se traduit pour le site hyperfin en question par deux solutions possibles présentant des valeurs principales et des directions principales différentes. Il ne faut guère compter sur l'inégalité précédente pour lever l'ambiguïté : les éléments non diagonaux ne sont généralement pas suffisamment grands pour que, dans l'alternative de signe qui joue sur le terme 2def l'inégalité ne soit vérifiée que dans un cas. Il n'est toutefois pas exclu d'espérer lever ultérieurement cette ambiguïté, comme déjà noté pour celles répertoriées au paragraphe 2 , par la comparaison avec un modèle théorique de tenseur.

Nous pouvons rencontrer le cas particulier accidentel où au moins l'élément non diagonal $f$ est nul et donc aussi le terme def. Alors, dans le plan correspondant, il existe une seule courbe d'extrema situés dans les directions des deux axes du repère abc que renferme ce plan. L'équation aux valeurs propres est unique et conduit donc à une seule solution : l'ambiguïté ne se pose pas ici. Remarquons que dans le cas du système monoclinique l'élément diagonal nul pourrait être celui lié au plan perpendiculaire à l'axe binaire ; bien qu'alors le nombre de façons d'associer les courbes dans les autres plans serait toujours de quatre, l'équation aux valeurs propres serait unique 
et l'ambiguïté ne se poserait pas non plus ici, étant entendu qu'à ce stade, il importe peu que les directions principales ne soient définies qu'à une indétermination globale près des signes des trois cosinus directeurs reliés à un même axe.

Le cas envisagé dans le sous-paragraphe précédent peut ne pas être fortuit : cela arrive si le radical présente une symétrie interne dont le groupe des rotations propres coïncide avec celui du cristal ou celui d'un de ses sous-groupes et si de plus cette symétrie conserve l'interaction hyperfine (nous savons que la symétrie peut aussi transformer une interaction hyperfine en une autre). Si le groupe est celui du cristal, le site hyperfin est unique : dans le cas monoclinique les deux éléments non diagonaux liés à l'axe binaire sont nuls et la direction de l'axe binaire est direction principale, dans le cas orthorhombique tous les éléments non diagonaux sont nuls et les directions des axes binaires sont les directions principales. Dans le cas orthorhombique, si la symétrie interne est limitée à l'un des axes binaires, il existe deux sites hyperfins dont les courbes sont distinctes seulement dans le plan perpendiculaire à l'axe binaire en question : les deux éléments non diagonaux liés à cet axe sont nuls et sa direction est principale.

Si l'on connaît les courbes des deux espèces de transitions dans les trois plans, l'ambiguïté est facilement levée. Seuls les bons jeux de signes, dans les matrices des deux espèces de tenseurs carrés, conduisent à une série commune de tenseurs $\mathbf{X}$ et c'est la solution physique recherchée pour la série de sites hyperfins. Il est d'autre part intéressant d'utiliser à titre complémentaire le procédé déjà vu qui consiste à obtenir la matrice de $\mathbf{X}$ directement par l'utilisation simultanée des deux espèces de transitions au moyen de la relation

$$
\mathbf{X}=\frac{1}{4 v}\left(\mathbf{A}_{v+}^{2}-\mathbf{A}_{v-}^{2}\right)
$$

Cette dernière méthode n'est pas suffisante en ellemême car elle entraîne ici une ambiguïté sur le signe de l'élément non diagonal du tenseur $\mathbf{X}$. De plus, elle donnerait deux valeurs absolues possibles pour un élément dans le cas où dans le plan correspondant on aurait quelque hésitation en ce qui concerne l'attribution des courbes de l'une et l'autre espèce au même site.

4. Regroupement des interactions hyperfines. Identification du radical. - Nous avons d'abord exposé le processus de calcul d'une interaction hyperfine détectée par ENDOR. Nous avons ensuite examiné les problèmes que soulevait dans l'expérience ENDOR puis dans le calcul de l'interaction l'existence de plusieurs sites pour le radical, en liaison avec les symétries que l'on trouve habituellement dans le cristal organique.

Nous avons rencontré plusieurs ambiguités qui entraînent l'existence de solutions parasites pour une interaction hyperfine. Ce sont dans l'ordre d'intervention dans le calcul :

a) l'alternative de signe pour l'élément non diagonal de la matrice d'un tenseur carré $\mathbf{A}^{2}$;

b) l'alternative de signe pour chaque valeur propre du tenseur simple $\mathbf{T}$;

c) l'ambiguité globale portant sur les signes absolus des valeurs propres du tenseur hyperfin $\mathbf{T}$.

Nous avons vu que les deux premières se résolvent plus ou moins complètement selon la richesse des résultats expérimentaux, ce n'est pas le cas de la troisième.

Nous avons implicitement résolu deux problèmes qui sont liés : l'identification des espèces complémentaires de transitions ENDOR (si on les détecte toutes deux) relevant d'une même interaction et l'identification de la nature du noyau impliqué dans cette interaction (même si c'est généralement un proton, il est quand même nécessaire de le vérifier). Dans ce but, alors que $v$ est encore inconnu, on néglige sa variation en fonction de $\mathbf{Z}$ pour avoir le droit de définir les divers tenseurs carrés. Il est alors aisé d'identifier les espèces complémentaires puisque leurs tenseurs carrés doivent avoir même vecteurs propres (ou plutôt dans ce calcul préliminaire des vecteurs propres très voisins). Il est ensuite possible d'évaluer $v$ à partir de :

$$
4 v \mathbf{X}=\mathbf{A}_{v+}^{2}-\mathbf{A}_{v-}^{2}
$$

et par exemple

$$
\mathbf{A}_{v+}^{2}=(\mathbf{X}+v)^{2},
$$

on obtient

$$
16 v^{4}-8 v^{2}\left(\mathbf{A}_{v+}^{2}+\mathbf{A}_{v-}^{2}\right)+\left(\mathbf{A}_{v+}^{2}-\mathbf{A}_{v-}^{2}\right)^{2}=0 .
$$

Cette équation, explicitée dans deux directions, fournit une valeur approchée pour $v$. Cette valeur approchée de $v$ associée à la valeur du champ magnétique permet d'identifier le noyau et donc la valeur exacte de $v$ pour cette valeur de champ magnétique.

En fait, dans la pratique nous sommes souvent dans le cas développé à la fin du paragraphe 1.3 où l'interaction hyperfine est suffisamment peu anisotrope pour que les courbes complémentaires soient plus ou moins symétriques par rapport à $v$ ou décalées d'environ $2 v$ : la résolution des deux problèmes est alors évidente. On peut éventuellement vérifier que $|\Delta m| \Delta v \mid \# 1$ en se plaçant à deux endroits du spectre R. P. E. : Il suffit de mesurer $\Delta m$ et de calculer $\Delta v$ pour la variation correspondante $\Delta H$ du champ magnétique.

Si par contre l'on ne détecte qu'une seule espèce de transitions ENDOR pour une interaction, on peut mesurer dans une direction $\mathbf{Z}$ la variation $\Delta m$ qui résulte d'une variation $\Delta H$. En supposant $|\Delta m / \Delta v| \not 1$, de $|\Delta v| \#|\Delta m|$ on déduit $v$. Si cette valeur de $v$ 
se trouve correspondre à la fréquence de résonance d'un certain noyau dans le champ magnétique $\mathbf{H}$, cette coïncidence ne peut guère que conduire à l'exactitude de la relation $|\Delta m| \Delta v \mid \not 1$ et le noyau se trouve par là même identifié. Notons que, d'après le paragraphe 1.3 , le signe de $\Delta m / \Delta H$ pourra éventuellement permettre de savoir si la transition est de fréquence haute ou de fréquence basse. $\mathrm{Si}$, toutefois, il reste quelque ambiguité sur la nature de ce noyau, on peut espérer la résoudre au cours de l'identification du radical.

Nous en arrivons alors au stade ultime où les interactions hyperfines, après calcul et recelant encore plus ou moins les ambiguïtés $a, b, c$, sont rassemblées pour être attribuées à des noyaux dont les positions auront à être bien précisées au cours de l'identification $\mathrm{du}$ radical.

\section{Il arrive à ce niveau deux problèmes :}

d) l'identification des couplages appartenant à un même radical, puisque c'est souvent que l'irradiation d'un cristal organique crée plusieurs radicaux : leurs spectres R. P. E. sont généralement mélangés ce qui fait que leurs raies ENDOR sont souvent simultanément obtenues. On peut espérer avoir classé les interactions des différents radicaux par l'aspect de leurs raies ENDOR respectives dont les largeurs et plus encore les intensités peuvent être différentes de manière caractéristique. En particulier, on aura pu rechercher dans le spectre $R$. P. E. des positions où les intensités des raies ENDOR, d'un radical à un autre, sont particulièrement contrastées ;

e) après le calcul d'un tenseur hyperfin $\mathbf{T}$, il existe une ambiguïté dans l'association du tenseur et du radical correspondant ou plus exactement de la molécule dont le radical dérive et dont il a gardé généralement, tout au moins dans les grandes lignes, la structure et la position dans le cristal. Bien entendu cela suppose qu'une étude cristallographique de la substance initiale a été par ailleurs faite. Le problème est simple: les trois axes cristallographiques abc sont généralement distinguables, mais on ne peut toutefois définir de manière unique un repère direct abc : il en existe quatre possibles. Possédant le positionnement de la molécule dans un repère direct abc, possédant d'autre part le tenseur hyperfin par ses valeurs propres et vecteurs propres dans un autre repère direct abc dont on ignore la position relative par rapport au premier, quatre solutions s'offriront finalement pour le tenseur: celle de départ et les trois qui s'en déduisent par l'un des trois changements globaux possibles des signes des cosinus directeurs correspondant à un même axe. Nous noterons que cette ambiguïté n'a rien à voir avec l'existence de sites bien que dans le cas du système orthorhombique cela revienne à se demander lequel parmi les quatre sites hyperfins l'on doit attribuer au site cristallographique sous considération. Elle existera tout autant s'il n'y a qu'un seul site.
Une possibilité très intéressante peut s'offrir expérimentalement de simplifier la levée des ambiguïtés $a$ et $e$. Si on arrive expérimentalement dans chaque plan d'étude à identifier les courbes des diverses interactions hyperfines d'un radical qui appartiennent à un même site hyperfin, chacune de ces ambiguités se posera ultérieurement une seule fois globalement et non plus dans chaque cas d'interaction hyperfine. Il suffirait ensuite de la résoudre dans le cas d'une interaction pour qu'elle soit simultanément résolue pour toutes les autres interactions. Pour classer les raies des différents sites, il est intéressant de rechercher dans chaque plan une position d'un spectre R.P. E. où les intensités des raies ENDOR de sites différents accuseraient une différence notable. Ce ne sera pas toujours facile puisque nous savons que dans bien des cas les couplages sont suffisamment peu anisotropes pour que les raies R. P. E. des différents sites hyperfins se chevauchent, conduisant à l'obtention simultanée des raies ENDOR de tous les sites.

Nous allons maintenant dire quelques mots $\mathrm{du}$ processus d'identification du radical, qui n'est évidemment pas propre à l'ENDOR, mais nous savons que seule l'ENDOR permet des investigations très poussées. L'identification du radical revêt deux aspects : identification de sa structure géométrique et identification de sa structure électronique. Lors de l'identification de la structure géométrique on attribue les interactions à leurs noyaux respectifs, ce qui permet de préciser les modifications subies par la molécule initiale lors de son passage à l'état de radical. Chaque tenseur s'apparente plus ou moins à l'un des divers modèles théoriques de tenseur d'interaction entre un électron dans une orbitale atomique et un noyau : cela fournit souvent une image géométrique grossière du système électron-noyau correspondant qui permet d'identifier la nature de l'orbitale atomique renfermant la fraction de densité de spin dont l'influence est prépondérante dans l'interaction hyperfine sous étude et qui permet de préciser les positions relatives de l'orbitale en question et du noyau. Nous savons d'autre part que généralement le radical conserve la place de la molécule dans la structure cristallographique. Il s'avère alors particulièrement fructueux de pouvoir disposer, au moyen d'une étude cristallographique, de la structure et du positionnement dans le cristal de la molécule initiale. Si tel est le cas, la localisation précise des divers systèmes électron-noyau par rapport à la molécule initiale peut être faite par comparaison des directions principales tensorielles et de directions de la molécule (direction des liaisons, direction de la normale au plan moléculaire dans le cas d'une molécule plane, etc...) permettant ainsi la détection des modifications survenues entre la molécule et le radical. Dans un deuxième stade une étude plus quantitative permet de reconstituer les tenseurs et d'accéder à la structure électronique du radical ou plus exactement à la carte de la 
densité de spin. Notons que la distinction entre les deux stades n'est pas aussi nette que nous l'écrivons.

En arrivant au stade de l'identification du radical, il reste de toute manière les ambiguïtés $c$ et $e$. Toutefois l'ambiguïté $e$ pourrait avoir été globalisée par ENDOR comme indiqué ci-dessus et, si tel est le cas, considérablement simplifiée. Il peut rester partiellement le problème $d$. Les ambiguïtés $a$ et $b$ sont totalement ou seulement partiellement résolues. Finalement, on sera donc en présence d'un problème plus ou moins complexe qui pourra entraîner plusieurs possibilités quant au radical. Mais nous devons remarquer que les considérations théoriques, les hypothèses et les analogies utilisées au cours de l'identification pourront lever de manière partielle si ce n'est complète les diverses ambiguités restantes avec la force d'une preuve. On peut en trouver des exemples concrets dans l'étude ENDOR du cristal de 1,2,4-Triazole irradié aux gammas [2, 6].

\section{Bibliographie}

[1] Farach, H. A. et Poole, Ch. P., Jr, Advances in Magnetic Resonance, édité par J. S. Waugh (Academic Press, New York et Londres) 5 (1971) p. 229 à 303.

[2] Gloux, P., Mol. Phys. 21 (1971) 829.

[3] RaE, A. D., J. Chem. Phys. 50 (1969) 2672.
[4] WeIL, J. A., Buch, T. et Clapp, J. E., Advances in Magnetic Resonance, édité par J. S. Waugh (Academic Press, New York et Londres) 6 (1973) p. 183 à 257.

[5] KitaigorodskiI, A. I., Organic Chemical Crystallography (1961).

[6] Gloux, P. et Lamotte, B., Mol. Phys. 25 (1973) 161. 